\title{
The Bed Nucleus of the Stria Terminalis Mediates Inter-individual Variations in Anxiety and Fear
}

\author{
Sevil Duvarci, ${ }^{\star}$ Elizabeth P. Bauer, ${ }^{\star}$ and Denis Paré \\ Center for Molecular and Behavioral Neuroscience, Rutgers, The State University of New Jersey, Newark, New Jersey 07102
}

While learning to fear stimuli that predict danger promotes survival, the inability to inhibit fear to inappropriate cues leads to a pernicious cycle of avoidance behaviors. Previous studies have revealed large inter-individual variations in fear responding with clinically anxious humans exhibiting a tendency to generalize learned fear to safe stimuli or situations. To shed light on the origin of these inter-individual variations, we subjected rats to a differential auditory fear conditioning paradigm in which one conditioned auditory stimulus (CS+) was paired to footshocks whereas a second (CS-) was not. We compared the behavior of rats that received pretraining excitotoxic lesions of the bed nucleus of the stria terminalis (BNST) to that of sham rats. Sham rats exhibit a continuum of anxious/fearful behaviors. At one end of the continuum were rats that displayed a poor ability to discriminate between the CS + and CS -, high contextual freezing, and an anxiety-like trait in the elevated plus maze (EPM). At the other end were rats that display less fear generalization to the CS -, lower freezing to context, and a nonanxious trait in the EPM. Although BNST-lesioned rats acquired similarly high levels of conditioned fear to the $\mathrm{CS}+$, they froze less than sham rats to the CS-. In fact, BNST-lesioned rats behaved like sham rats with high discriminative abilities in that they exhibited low contextual fear and a nonanxious phenotype in the EPM. Overall, this suggests that inter-individual variations in fear generalization and anxiety phenotype are determined by BNST influences on the amygdala and/or its targets.

\section{Introduction}

The neural substrates of Pavlovian fear conditioning continue to attract much interest, in part because human subjects with anxiety disorders exhibit abnormalities in conditioned fear (Grillon, 2002; Milad et al., 2008; Lissek et al., 2009). While it remains controversial whether anxiety disorders represent pathological manifestations of normal fear-learning mechanisms (McNally, 2002; Mineka and Ohman, 2002; Poulton and Menzies, 2002; Mineka and Oehlberg, 2008), there is consensus that the structures normally involved in such learning display abnormal activity patterns in anxious subjects (Shin et al., 2006; Bremner et al., 2008). Thus, understanding the neural alterations underlying inter-individual variations in conditioned fear may yield fundamental insights into the causes of anxiety disorders.

Rodent studies have documented intersubject heterogeneity in the acquisition and extinction of conditioned fear responses. In some instances, these variations were seen between subjects of the same strain (Milad and Quirk, 2002; Burgos-Robles et al., 2007). In other cases, they emerged after selective breeding for particular behavioral properties (Brush et al., 1999) or manifested themselves in different strains of rats or mice (Cohen et al., 2006; Hefner et al., 2008). In the present study, we focused on

\footnotetext{
Received May 5, 2009; revised July 7, 2009; accepted July 17, 2009.

This work was supported by National Institutes of Health R01 Grant MH-083710 to D.P. and National Research Service Award Grant 5F32MH076640 to E.P.B.

*S.D. and E.P.B. contributed equally to this work.

Correspondence should be addressed to Denis Paré, Center for Molecular and Behavioral Neuroscience, Rutgers, The State University of New Jersey, 197 University Avenue, Newark, NJ 07102. E-mail: pare@androemda.rutgers.edu. DOI:10.1523/JNEUROSCI.2119-09.2009

Copyright $\odot 2009$ Society for Neuroscience $\quad$ 0270-6474/09/2910357-05\$15.00/0
}

Lewis rats, an inbred strain that displays marked interindividual variations in contextual and cued fear conditioning. To probe the neural origins of these variations, we used a fear conditioning task that allowed us to simultaneously assess the influence of two components of the brain circuitry regulating fear and anxiety: the amygdala and bed nucleus of the stria terminalis (BNST).

Indeed, previous behavioral studies have revealed that the amygdala plays a critical role in the acquisition and immediate expression of conditioned fear responses to discrete sensory cues (Hitchcock and Davis, 1987, 1991; LeDoux et al., 1988; Kim et al., 1993; Campeau and Davis, 1995; Wilensky et al., 2006), behaviors that are not affected by BNST lesions (LeDoux et al., 1988; Walker and Davis, 1997; Gewirtz et al., 1998; Sullivan et al., 2004). Instead, BNST lesions were reported to prevent the development of longer "anxiety-like" states in response to more diffuse environmental contingencies (for review, see Walker et al., 2003). Yet, there are strong reciprocal connections between these two structures (Krettek and Price, 1978b; Price and Amaral, 1981; Sun and Cassell, 1993; Veinante and Freund-Mercier, 2003; Dong et al., 2001; Dong and Swanson, 2006a,b,c), suggesting that their interactions determine behavioral output.

Reasoning that BNST influence might not manifest itself in the magnitude of conditioned fear responses to cues but in more subtle measures, such as stimulus specificity and modulation by context, the present study examined inter-individual variations along these dimensions and probed the impact of BNST lesions. Overall, our results suggest that BNST influences over the amygdala and/or its targets shape inter-individual variations in the expression of fear and anxiety. 


\section{Materials and Methods}

Subjects. In the present study, we used Lewis rats because a pilot experiment involving the same protocol as described below revealed much more inter-individual heterogeneity in this inbred strain than in the more commonly used Sprague Dawley rats. Lewis rats (adult males, Charles River Laboratories) were housed individually with ad libitum access to food and water and maintained on a $12 \mathrm{~h}$ light/dark cycle. All procedures were conducted in accordance with the National Institutes of Health Guide for the Care and Use of Laboratory Animals and were approved by the Rutgers University Animal Care and Use Committee.

Surgery. Rats were anesthetized with a mixture of isoflurane and oxygen and were administered atropine methyl nitrate to reduce secretions and aid breathing. In aseptic conditions, rats were mounted in a stereotaxic apparatus with nonpuncture ear bars. Betadine was applied to the scalp, and a local anesthetic (bupivacaine, s.c.) was injected in the region to be incised. Ten minutes later, the scalp was incised, and small burr holes were made in the skull above the BNST. Bilateral BNST lesions were made by injecting $0.6 \mu \mathrm{l}$ (per side) of ibotenic acid (Sigma) dissolved in artificial CSF $(6 \mu \mathrm{g} / \mu \mathrm{l})$. This total volume was stereotaxically (Paxinos and Watson, 1998) infused at two different anteroposterior levels of the BNST $(0.3 \mu \mathrm{l}$ at each site). Injections were made at a rate of $0.1 \mu \mathrm{l} / \mathrm{min}$ with a Hamilton microsyringe. After the injection, the wound was sutured and a local antibiotic applied. Rats were given at least 1 week to recover before the beginning of behavioral procedures.

Differential fear conditioning paradigm. Fear conditioning and recall testing occurred in different contexts (contexts A and B). For fear conditioning (context $\mathrm{A}$ ), rats were placed in a rodent conditioning chamber with a metal grid floor (Coulbourn Instruments) that was enclosed within a sound attenuating chamber. The chamber was dimly illuminated by a single house light. For testing recall, the chamber contained a black Plexiglas floor washed with peppermint soap (context B). Figure $1 \mathrm{~A}$ summarizes the paradigm. On day 0 , rats were habituated to contexts $\mathrm{A}$ and $\mathrm{B}$ for 20 min each in a counterbalanced manner. This was followed by tone habituation sessions in each context in which rats received five presentations of two different auditory stimuli (white noise or $2 \mathrm{kHz}, 80$ $\mathrm{dB}, 30 \mathrm{~s}$ duration) in a counterbalanced manner and random order. On day 1 , rats were placed in context $A$ and subjected to a differential fear conditioning paradigm in which one of the two auditory stimuli listed above served as conditioned stimulus ( $\mathrm{CS}+$ ) because it was paired to a footshock, whereas the second conditioned stimulus (CS-) was not. During fear conditioning, the animals received 5 CS + and 5 CS - in a random order. The CS+ coterminated with a footshock unconditioned stimulus $(0.5 \mathrm{~mA}, 1 \mathrm{~s})$. On day 2 , rats were placed in context A for $10 \mathrm{~min}$ to test for contextual fear memory. On day 3 , in context B, recall was tested with additional presentations of the CS + and CS - . Behavior was recorded by a video camera and scored offline by an experienced observer blind to the rats' identity. Time spent freezing (immobility, with the exception of breathing) was measured during the context or CS presentations for each rat. Two days after the recall test, rats were placed in an elevated plus maze for $5 \mathrm{~min}$ to assess anxiety levels. The time spent in the center and on the open and closed arms was then scored offline.

Histology. At the end of behavioral experiments, the animals were given an overdose of pentobarbital $\left(100 \mathrm{mg} \cdot \mathrm{kg}^{-1}\right.$, i.p. $)$ and perfused transcardially with $0.9 \%$ saline, followed by paraformaldehyde $(4 \%)$. The brains were then removed, stored in paraformaldehyde (4\%), sectioned at a thickness of $100 \mu \mathrm{m}$, and processed to reveal NeuN immunoreactivity as previously described (Koo et al., 2004). Lesion extent was verified by reconstructing the damage on stereotaxic atlas templates (Paxinos and Watson, 1998). Acceptable lesions included bilateral damage of the BNST throughout most of its extent with minor damage of surrounding areas.

\section{Results}

The experimental paradigm used in the present study is shown in Figure $1 A$. Lewis rats received vehicle $(n=28)$ or ibotenic acid infusions $(n=40)$ aimed to the BNST and were allowed to recover for 1 week. BNST-lesioned and sham rats then underwent the same protocol in parallel. After habituation (Fig. $1 \mathrm{~A}$, day 0 ),
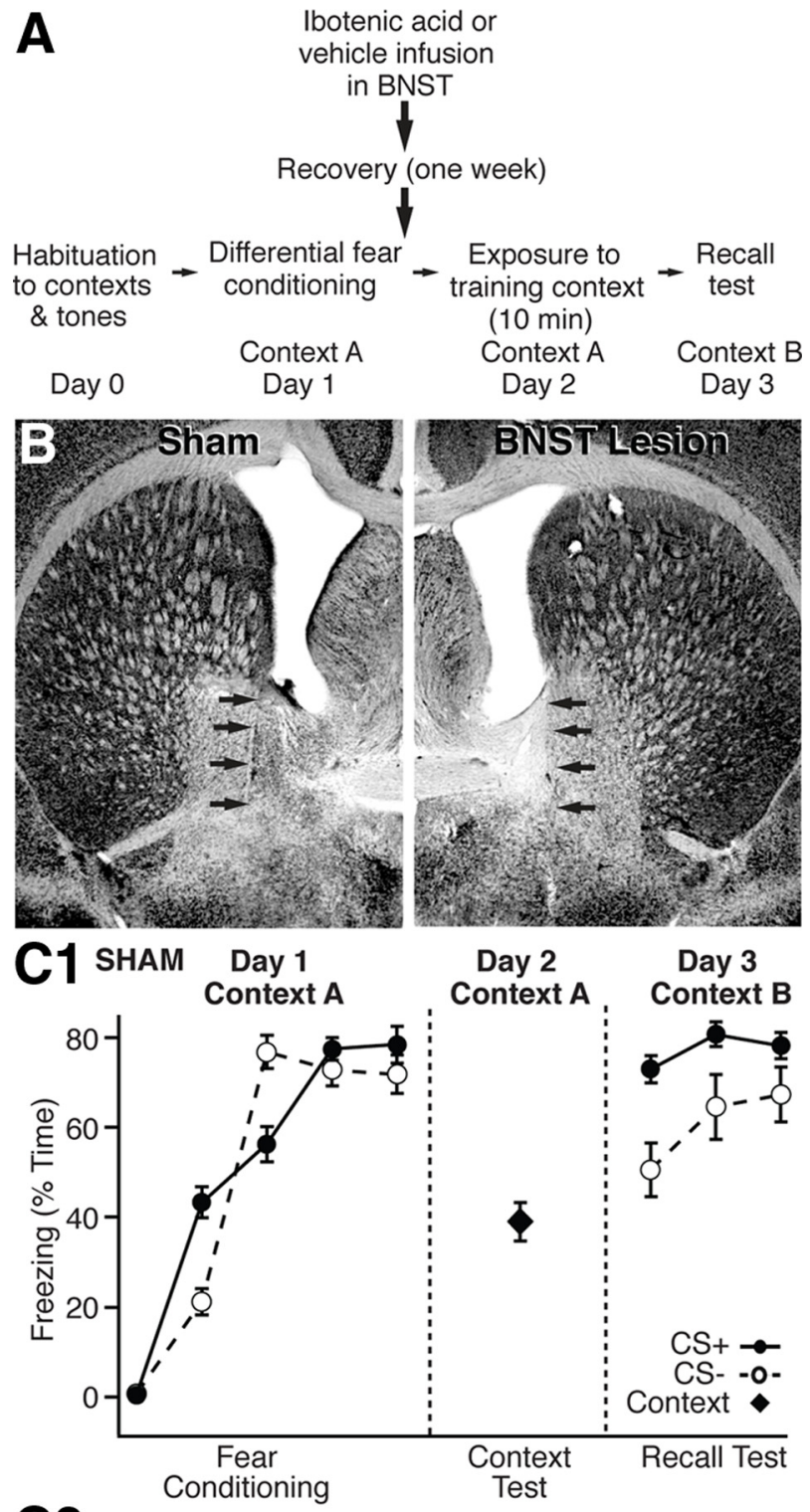

C2 BNST Lesion

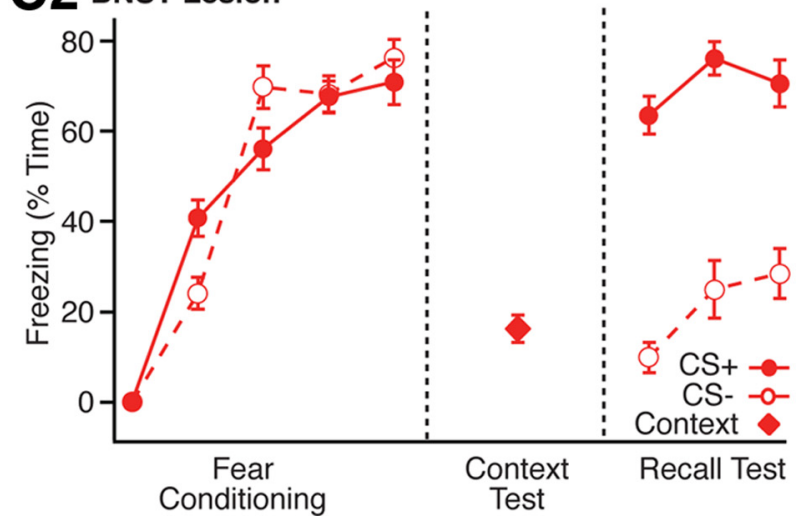

Figure 1. Differential auditory fear conditioning in sham versus BNST-lesioned rats. $A$, Experimental paradigm. $\boldsymbol{B}$, NeuN immunoreactivity in coronal sections of sham (left) versus BNSTlesioned rats (right). $C$, Percentage time freezing (average \pm SEM) in response to the $C S+$ (filled circles) and CS - (empty circles) during fear conditioning (left), during exposure to the training context without tones (middle), and during the recall test (right) in sham $(n=28$; C1, black) versus BNST-lesioned $(n=18 ; \mathbf{C}$, red) rats. 
A
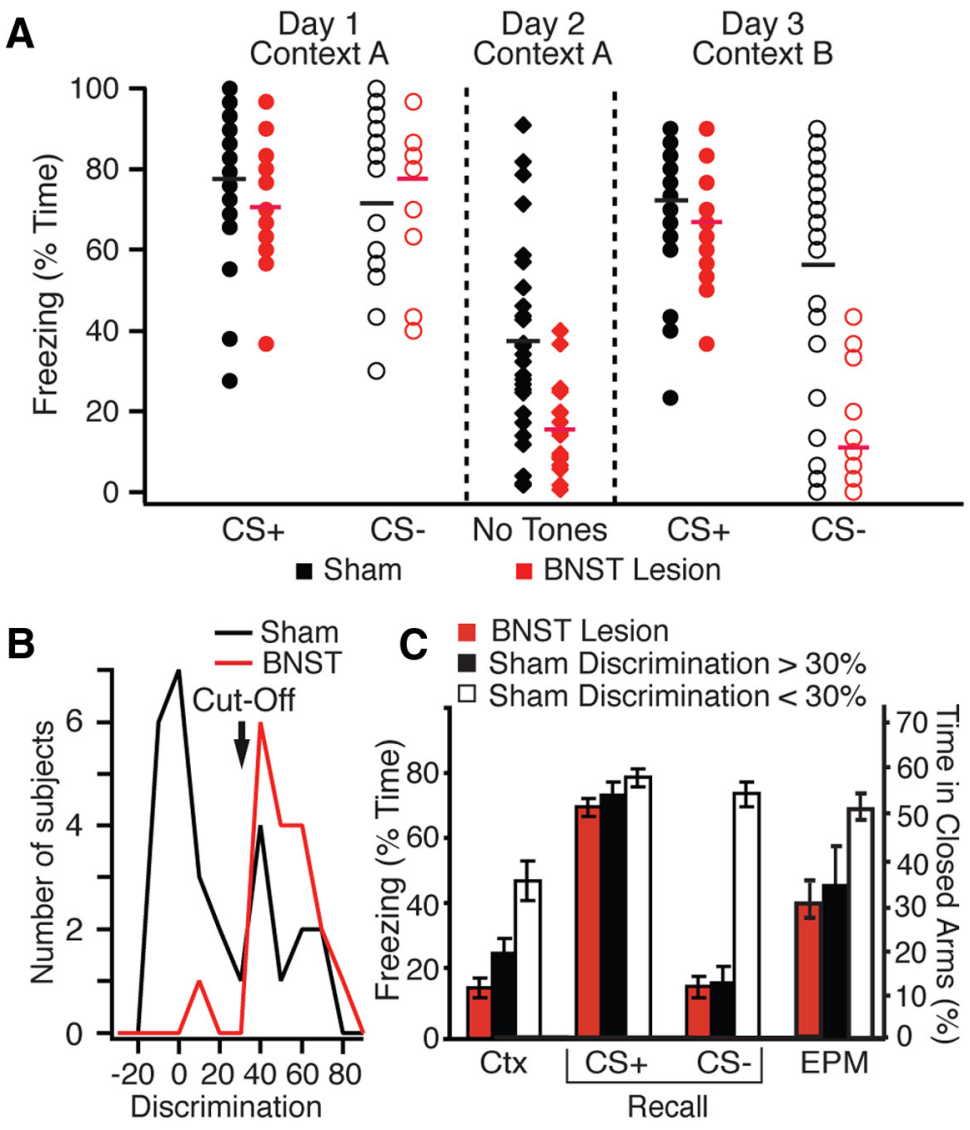

C BNST Lesion

C Sham Discrimination $>30 \%$
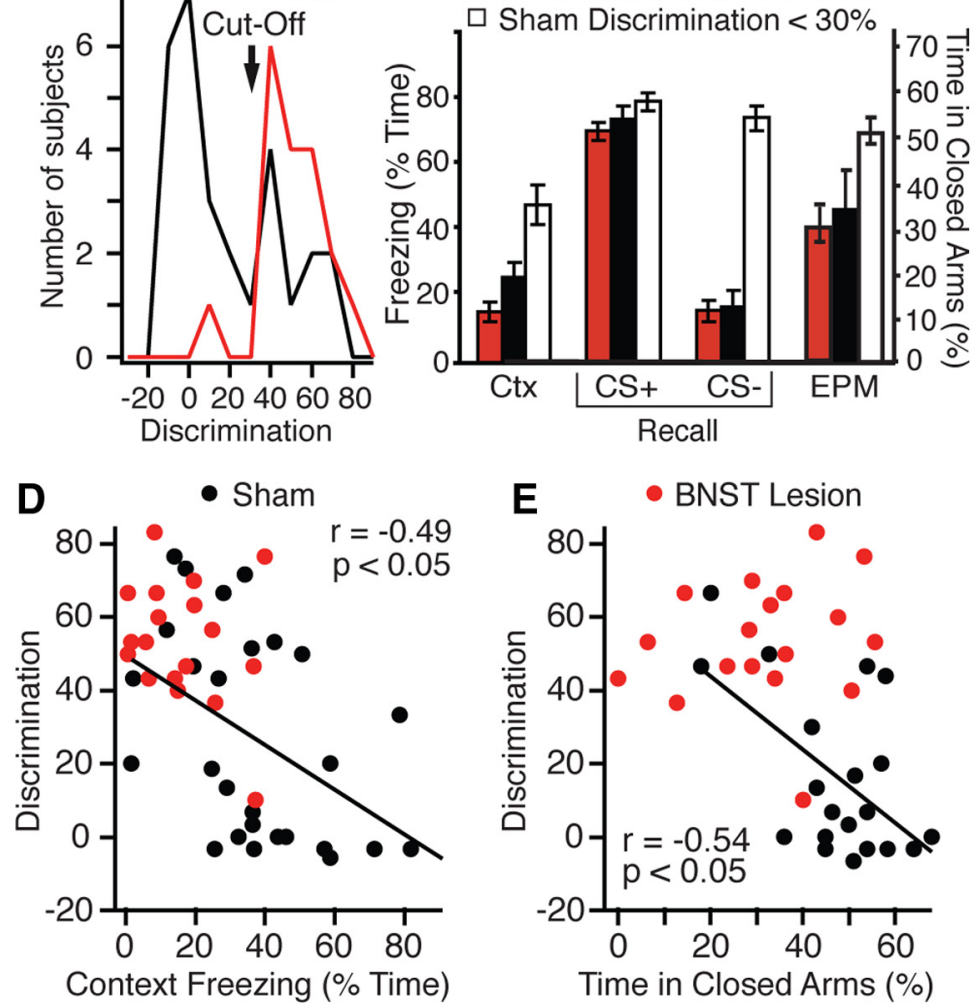

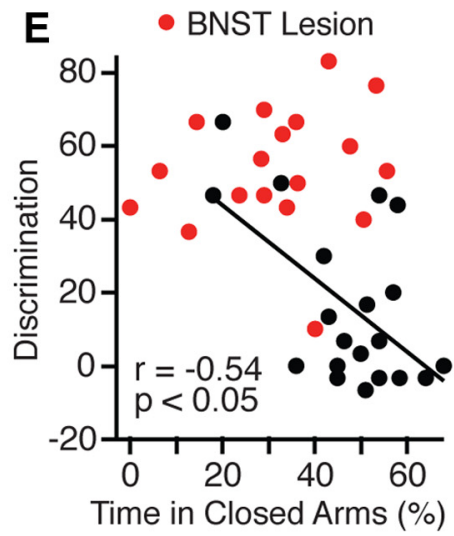

Figure 2. BNST lesions abolish behavioral heterogeneity related to discrimination abilities. $\boldsymbol{A}$, Percentage time freezing in response to the $C S$ - (filled circles) and $C S$ - (empty circles) in individual sham (black; $n=28$ ) versus BNST-lesioned (red; $n=18$ ) rats during fear conditioning (left), during exposure to the training context without tones (middle), and during the recall test (right). Lines indicate averages. B, Frequency distribution of discrimination scores in sham (black) versus BNST-lesioned (red) rats. C, Comparison between BNST-lesioned rats (red; $n=18$ ) and sham rats with high (black bars; $n=10$ ) or low (white bars; $n=18$ ) discrimination scores in percentage time freezing (left $y$-axis) to training context (Ctx) and to $C S+$ and $C S$ - during the recall test, as well as time in closed arms of EPM (right $y$-axis). D, E, Discrimination score ( $y$-axis) versus freezing to training context ( $x$-axis, D) or time in closed arms $(x$-axis, $\boldsymbol{E})$.

they were subjected to a differential fear conditioning paradigm (Fig. $1 A$, day 1 ) in which one of two tones (CS+) was paired to a footshock whereas a second tone (CS-) was not. On day 2, freezing to the context was assessed. Recall of cued fear was tested on day 3 in a different context. Two days after the recall test, anxiety levels were assessed using an elevated plus maze (EPM). At the conclusion of the experiments, the animals were perfused-fixed, their brains sectioned, and the sections processed to reveal NeuN immunoreactivity (Fig. $1 B$ ) to assess the location and specificity of the lesions. On this basis, we restricted our attention to the behavior of a subset of 18 animals that had extensive and selective BNST lesions. See supplemental Figure 1 (available at www.jneurosci.org as supplemental material) for the extent of the largest and smallest BNST lesions included in this study.

Figures 1 and 2 illustrate in parallel the results obtained in sham (black lines and symbols) and BNST-lesioned (red lines and symbols) rats. Filled and empty circles represent freezing to the CS+ and CS-, respectively. Diamonds represent contextual freezing in the absence of CS. To facilitate explanation of the data, we first describe the results obtained in sham rats and then contrast their behavior to that of BNST-lesioned animals.

Sham rats exhibited normal acquisition of conditioned fear responses to the CS + (day 1), significant freezing to the training context (day 2), and excellent fear recall (day 3) (Fig. 1C1). Although they exhibited poor discrimination between the CS+ and CS- during conditioning and recall, freezing to the CS- was significantly lower than to the CS+ during recall ( $t$ test, first CS + vs CS,$- p=0.001)$. However, there was considerable interindividual heterogeneity in fear responding among sham rats, particularly during recall (Fig. $2 \mathrm{~A}$, black). Although all sham rats exhibited high freezing in response to the $\mathrm{CS}+$, freezing to the CS - was widely distributed, with some animals freezing as much to the CS - as to the CS+, and others freezing much less to the CS-. Thus, although the collective behavior of sham rats indicates poor discrimination between the CS+ and CS-, inter-individual variations suggest a more complex situation in which some subjects exhibit good discrimination between the two CSs, whereas others generalize fear to the safe tone (CS-).

Next, we determined whether the varying aptitude to discriminate between the two CSs seen in sham rats was associated with other behavioral properties. To this end, we computed a frequency distribution of the difference in freezing to the first CS + and CS - of the recall test, hereafter termed discrimination score (DS), operationally defined as a measure of discriminative ability. The ability to discriminate between the two CSs was not distributed normally among sham rats, the frequency distribution being highly skewed to the right (Fig. $2 \mathrm{~B}$, black). On the basis of this distribution, we used a cutoff of $30 \%$ difference in discrimination score to distinguish sham rats with low versus high discriminative abilities.

No difference in the amount of freezing to the CS + was found between the two subsets of sham rats during recall (Fig. $2 C)(p>$ 
0.05; $t$ test comparing freezing to the first CS + ). In contrast, sham rats with a high discrimination score exhibited significantly less freezing to the training context (Fig. 2C, Ctx) $(24.0 \pm 4.4 \%)$ than poor discriminators $(45.1 \pm 5.4 \%, p=0.03)$. In keeping with this, discrimination score and context freezing were inversely correlated among sham rats (Fig. 2D) $(r=-0.49, p<$ 0.05). Moreover, poor discriminators spent significantly more time in the closed arms of the EPM than sham rats with good discriminative abilities (Fig. 2C, EPM) ( $t$ test, $p=0.03$ ), these two variables being inversely correlated (Fig. $2 E)(r=-0.54, p<$ $0.05)$.

Thus, the above analysis suggests that sham rats exhibit a continuum of anxious/fearful behaviors where different measures of fear and anxiety tend to covary. At one pole of the distribution are rats that display poor discriminative abilities, high contextual freezing, and a marked anxiety-like trait in the EPM. At the other pole are rats that display less fear generalization to the CS-, lower freezing to context, and a nonanxious trait on the EPM. See supplemental Figure 2 (available at www.jneurosci.org as supplemental material) for examples.

Insights into BNST contributions to these inter-individual variations can be gained by comparing the behavior of sham versus BNST-lesioned rats. Since the ability to discriminate between the CS + and CS - during recall was most predictive of fearful/anxious behavior in sham rats, we first compare the two groups along this dimension. Discrimination ability during the recall test was drastically higher in rats with BNST lesions (Figs. $1 C 2,2 \mathrm{~A}$, red) than in sham rats (Figs. $1 C 1,2 \mathrm{~A}$, black). Indeed, 17 of 18 BNST-lesioned animals had a DS $>30 \%$ (Fig. $2 B$ ). The differing proportion of rats with low or high DSs in sham versus BNST-lesioned rats was significant (Fisher exact test, $p=$ $0.00006)$. So were the average DSs of sham $(21.7 \pm 4.9 \%)$ and BNST-lesioned rats $(53.7 \pm 3.9 \%$; $t$ test, $p=0.00004)$. This difference was due to decreased freezing to the CS - since behavior during the CS + did not differ between the two groups (Fig. 2C) $(t$ test, $p=0.52$ ).

Since discriminative ability was predictive of anxious/fearful behavior in the sham animals, we next examined whether BNSTlesioned animals behaved like sham rats with high DSs (Fig. 2C). In keeping with this, BNST-lesioned rats were statistically indistinguishable from sham rats with high discriminative abilities in the amount of contextual freezing they displayed (Fig. 2C) ( $t$ test, $p=0.14$ ) and time spent in the closed arms of the EPM ( $t$ test, $p=$ $0.45)$. The marked reduction in contextual freezing seen in rats with BNST lesions is consistent with previous reports (Sullivan et al., 2004; Resstel et al., 2008).

\section{Discussion}

The present study was undertaken to shed light on the origin of inter-individual variations in fear an anxiety. By comparing the behavior of sham versus BNST lesioned rats in a differential fear conditioning paradigm, we obtained evidence that interindividual variations in the expression of fear and anxiety are determined, at least in part, by BNST influences over its targets. In the following account, we consider the significance of these observations in light of earlier physiological and behavioral findings about the role of the amygdala and BNST.

\section{Dissociation between BNST and amygdala functions despite similar connectivity}

Previous behavioral studies have emphasized the contrasting roles of the BNST and central amygdala (CEA) in fear and anxiety (for review, see Walker et al., 2003). Lesion, stimulation, and pharmacobehavioral studies (for review, see Davis, 2000) suggest that CEA is the main output station of the amygdala for the rapid generation of brief conditioned fear responses to discrete sensory cues (however see Koo et al., 2004). These fear responses are mediated by divergent CEA projections to an array of hypothalamic and brainstem structures, such as the periaqueductal gray for freezing (Hopkins and Holstege, 1978; LeDoux et al., 1988). BNST lesions, however, do not disrupt conditioned fear to cues but contextual fear (LeDoux et al., 1988; Walker and Davis, 1997; Gewirtz et al., 1998; Sullivan et al., 2004). In fact, the available data suggests that BNST mediates slowly developing and longlasting responses to diffuse threats (for review, see Walker et al., 2003).

The contrasting role of CEA and BNST in fear and anxiety is puzzling because these two structures exhibit a similar connectivity. For instance, they both receive excitatory inputs from the basolateral amygdala (Krettek and Price, 1978a,b; Sun and Cassell, 1993; Dong et al., 2001), the two are reciprocally connected, and their brainstem projections overlap extensively (Hopkins and Holstege, 1978; Price and Amaral, 1981; Dong et al., 2000; Dong and Swanson, 2004, 2006a,b,c). Moreover, a recent physiological study revealed that the conduction time of BNST and CEA neurons allows for a synchronization of their brainstem outputs (Nagy and Paré, 2008).

Although consistent with previous lesion studies, the present report indicates that CEA and BNST functions are not entirely independent after all. Indeed, while BNST lesions did not affect the magnitude of conditioned fear responses to the CS+, they decreased inappropriate fear responding to the CS - . This finding suggests that even though BNST activity is not required for the generation of conditioned fear to cues, it determines its selectivity.

\section{Inter-individual variations in fear and anxiety}

There was marked inter-individual heterogeneity in the amount of fear and anxiety displayed by Lewis rats. Interestingly, different measures of fear and anxiety correlated with each other and with the ability to discriminate between the two CSs. Indeed, rats that exhibited high discriminative abilities tended to show low contextual fear and spent little time in the closed arms of the EPM. Conversely, rats that showed poor discriminative abilities displayed high levels of contextual fear and an anxious phenotype in the EPM. Remarkably, following BNST lesions, the anxious EPM phenotype was abolished, contextual fear was reduced, and the vast majority of rats exhibited high discriminative abilities.

These observations indicate that the poor discriminative abilities seen in a subset of sham Lewis rats are not due to a basic sensory deficit in differentiating between the two CSs. Rather, this suggests that at a subsequent processing step, probably downstream of auditory thalamocortical regions, the pattern of activity evoked by the two CSs fails to diverge in rats with the anxious/fearful phenotype. Currently, there is insufficient information to pinpoint where this occurs. However, the fact that BNST lesions restored the CS specificity of fear responding suggests that BNST and some of its outputs are involved.

This latter view is supported by a recent primate study in which individual variations in anxious temperament were predicted by presynaptic serotonin reuptake transporter binding (Shelton et al., 2008) and glucose uptake (Fox et al., 2008) in the BNST. Thus, it is likely that in subjects with the anxious/fearful phenotype, BNST neurons have higher baseline and/or sensorydriven levels of activity. These findings suggest that pharmaco- 
logical interventions that reduce BNST excitability may prove useful for the treatment of anxiety disorders.

\section{References}

Bremner JD, Elzinga B, Schmahl C, Vermetten E (2008) Structural and functional plasticity of the human brain in posttraumatic stress disorder. Prog Brain Res 167:171-186.

Brush FR, Gendron CM, Isaacson MD (1999) A selective genetic analysis of the Syracuse high- and low-avoidance (SHA/Bru and SLA/Bru) strains of rats (Rattus norvegicus). Behav Brain Res 106:1-11.

Burgos-Robles A, Vidal-Gonzalez I, Santini E, Quirk GJ (2007) Consolidation of fear extinction requires NMDA receptor-dependent bursting in the ventromedial prefrontal cortex. Neuron 53:871-880.

Campeau S, Davis M (1995) Involvement of subcortical and cortical afferents to the lateral nucleus of the amygdala in fear conditioning measured with fear-potentiated startle in rats trained concurrently with auditory and visual conditioned stimuli. J Neurosci 15:2312-2327.

Cohen H, Zohar J, Gidron Y, Matar MA, Belkind D, Loewenthal U, Kozlovsky N, Kaplan Z (2006) Blunted HPA axis response to stress influences susceptibility to posttraumatic stress response in rats. Biol Psychiatry 59:1208-1218.

Davis M (2000) The role of the amygdala in conditioned and unconditioned fear and anxiety. In: The amygdala: a functional analysis (Aggleton JP, ed), pp 213-287. Oxford: Oxford UP.

Dong H, Petrovich GD, Swanson LW (2000) Organization of projections from the juxtacapsular nucleus of the BST: a PHAL study in the rat. Brain Res 859:1-14.

Dong HW, Swanson LW (2004) Organization of axonal projections from the anterolateral area of the bed nuclei of the stria terminalis. J Comp Neurol 468:277-298.

Dong HW, Swanson LW (2006a) Projections from bed nuclei of the stria terminalis, dorsomedial nucleus: implications for cerebral hemisphere integration of neuroendocrine, autonomic, and drinking responses. J Comp Neurol 494:75-107.

Dong HW, Swanson LW (2006b) Projections from bed nuclei of the stria terminalis, magnocellular nucleus: implications for cerebral hemisphere regulation of micturition, defecation, and penile erection. J Comp Neurol 494:108-141.

Dong HW, Swanson LW (2006c) Projections from bed nuclei of the stria terminalis, anteromedial area: cerebral hemisphere integration of neuroendocrine, autonomic, and behavioral aspects of energy balance. J Comp Neurol 494:142-178.

Dong HW, Petrovich GD, Swanson LW (2001) Topography of projections from amygdala to bed nuclei of the stria terminalis. Brain Res Brain Res Rev 38:192-246.

Fox AS, Shelton SE, Oakes TR, Davidson RJ, Kalin NH (2008) Trait-like brain activity during adolescence predicts anxious temperament in primates. PLoS ONE 3:e2570.

Gewirtz JC, McNish KA, Davis M (1998) Lesions of the bed nucleus of the stria terminalis block sensitization of the acoustic startle reflex produced by repeated stress, but not fear-potentiated startle. Prog Neuropsychopharmacol Biol Psychiatry 22:625-648.

Grillon C (2002) Startle reactivity and anxiety disorders: aversive conditioning. Biol Psychiatry 52:958-975.

Hefner K, Whittle N, Juhasz J, Norcross M, Karlsson RM, Saksida LM, Bussey TJ, Singewald N, Holmes A (2008) Impaired fear extinction learning and cortico-amygdala circuit abnormalities in a common genetic mouse strain. J Neurosci 28:8074-8085.

Hitchcock JM, Davis M (1987) Fear-potentiated startle using an auditory conditioned stimulus: effect of lesions of the amygdala. Physiol Behav 39:403-408.

Hitchcock JM, Davis M (1991) Efferent pathway of the amygdala involved in conditioned fear as measured with the fear-potentiated startle paradigm. Behav Neurosci 105:826-842.

Hopkins DA, Holstege G (1978) Amygdaloid projections to the mesencephalon, pons and medulla oblongata in the cat. Exp Brain Res 32:529-547.

Kim M, Campeau S, Falls WA, Davis M (1993) Infusion of the non-NMDA receptor antagonist CNQX into the amygdala blocks the expression of fear-potentiated startle. Behav Neural Biol 59:5-8.

Koo JW, Han JS, Kim JJ (2004) Selective neurotoxic lesions of basolateral and central nuclei of the amygdala produce differential effects on fear conditioning. J Neurosci 24:7654-7662.

Krettek JE, Price JL (1978a) Amygdaloid projections to subcortical structures within the basal forebrain and brainstem in the rat and cat. J Comp Neurol 178:225-254.

Krettek JE, Price JL (1978b) A description of the amygdaloid complex in the rat and cat with observations on intra-amygdaloid axonal connections. J Comp Neurol 178:255-280.

LeDoux JE, Iwata J, Cicchetti P, Reis DJ (1988) Different projections of the central amygdaloid nucleus mediate autonomic and behavioral correlates of conditioned fear. J Neurosci 8:2517-2529.

Lissek S, Rabin SJ, McDowell DJ, Dvir S, Bradford DE, Geraci M, Pine DS, Grillon C (2009) Impaired discriminative fear-conditioning resulting from elevated fear responding to learned safety cues among individuals with panic disorder. Behav Res Ther 47:111-118.

McNally RJ (2002) On nonassociative fear emergence. Behav Res Ther 40:169-172.

Milad MR, Quirk GJ (2002) Neurons in medial prefrontal cortex signal memory for fear extinction. Nature 420:70-74.

Milad MR, Orr SP, Lasko NB, Chang Y, Rauch SL, Pitman RK (2008) Presence and acquired origin of reduced recall for fear extinction in PTSD: results of a twin study. J Psychiatr Res 42:515-520.

Mineka S, Oehlberg K (2008) The relevance of recent developments in classical conditioning to understanding the etiology and maintenance of anxiety disorders. Acta Psychol (Amst) 127:567-580.

Mineka S, Ohman A (2002) Born to fear: non-associative vs associative factors in the etiology of phobias. Behav Res Ther 40:173-184.

Nagy FZ, Paré D (2008) Timing of impulses from the central amygdala and bed nucleus of the stria terminalis to the brain stem. J Neurophysiol 100:3429-3436.

Paxinos G, Watson C (1998) The rat brain in stereotaxic coordinates. San Diego: Academic.

Poulton R, Menzies RG (2002) Non-associative fear acquisition: a review of the evidence from retrospective and longitudinal research. Behav Res Ther 40:127-149.

Price JL, Amaral DG (1981) An autoradiographic study of the projections of the central nucleus of the monkey amygdala. J Neurosci 1:1242-1259.

Resstel LB, Alves FH, Reis DG, Crestani CC, Corrêa FM, Guimarães FS (2008) Anxiolytic-like effects induced by acute reversible inactivation of the bed nucleus of stria terminalis. Neuroscience 154:869-876.

Shelton SE, Fox AS, Oler JA, Oakes TR, Christian BT, Davidson RJ, Kalin NH (2008) Individual differences in anxious temperament are predicted by 5 -HTT binding in the bed nucleus of the stria terminalis. Soc Neurosci Abstr 34:256.4.

Shin LM, Rauch SL, Pitman RK (2006) Amygdala, medial prefrontal cortex, and hippocampal function in PTSD. Ann N Y Acad Sci 1071:67-79.

Sullivan GM, Apergis J, Bush DE, Johnson LR, Hou M, Ledoux JE (2004) Lesions in the bed nucleus of the stria terminalis disrupt corticosterone and freezing responses elicited by a contextual but not by a specific cueconditioned fear stimulus. Neuroscience 128:7-14.

Sun N, Cassell MD (1993) Intrinsic GABAergic neurons in the rat central extended amygdala. J Comp Neurol 330:381-404.

Veinante P, Freund-Mercier MJ (2003) Branching patterns of central amygdaloid nucleus efferents in the rat: single-axon reconstructions. Ann N Y Acad Sci 985:552-553.

Walker DL, Davis M (1997) Double dissociation between the involvement of the bed nucleus of the stria terminalis and the central nucleus of the amygdala in startle increases produced by conditioned versus unconditioned fear. J Neurosci 17:9375-9383.

Walker DL, Toufexis DJ, Davis M (2003) Role of the bed nucleus of the stria terminalis versus the amygdala in fear, stress, and anxiety. Eur J Pharmacol 463:199-216.

Wilensky AE, Schafe GE, Kristensen MP, LeDoux JE (2006) Rethinking the fear circuit: the central nucleus of the amygdala is required for the acquisition, consolidation, and expression of pavlovian fear conditioning. J Neurosci 26:12387-12396. 